\title{
Hardware-efficient auto-reconfigurable hearing aids using 3-level octave interpolated filters for auditory compensation applications
}

\author{
Tomson Devis ${ }^{1}$ (1) $\cdot$ Manju Manuel ${ }^{2}$
}

Received: 25 October 2020 / Accepted: 22 June 2021 / Published online: 6 July 2021

๑) Australasian College of Physical Scientists and Engineers in Medicine 2021

\begin{abstract}
A reconfigurable hearing aid is a generic type that can be used for various hearing disabilities without modifying the device hardware. This requires several trials to identify the best matching with the impaired person's audiogram. The objective of this paper is to propose a novel reconfigurable hearing aid of low complexity with auto-adapting capability which makes it suitable for different types of hearing disabilities ranging from mild to severe intensities. The audio spectrum is divided into three regions and for each region, four different schemes are proposed. An automatic selection of the optimum scheme is proposed for all the regions based on hearing thresholds. Octave and fractional interpolation techniques are performed on a Parks-McClellan based prototype filter to generate the various sub-bands in the reconfigurable filter bank structure. The proposed structure uses only 18 coefficient multipliers which save up to $92 \%$ of multipliers when compared to other designs. The delay and matching errors are within the globally accepted limits. The hardware implementation executed on Xilinx Kintex-7 FPGA development board has reaffirmed that the structure is compact and power-efficient. The proposed auto-reconfigurable structure can be used for various types of hearing impairments and can avoid the manual interventions for the selection of schemes in audiogram matching. This in turn minimizes the time to establish the best match with the audiogram. Since the proposed structure has minimal complexity, cost-effective implementation of the device is also possible.
\end{abstract}

Keywords Multirate system $\cdot$ Filter bank $\cdot$ Hearing aid $\cdot$ Auto-reconfigurable $\cdot$ Audiogram $\cdot$ Matching error

\section{Introduction}

Digital hearing aids remain the most popular type of hearing assistive device due to its improved performance and efficient implementation [1]. They have the advantage of enhanced programmability which makes them suitable for being customized by the requirements of hearing-impaired individuals [2]. Digital hearing aids are capable of performing complex signal processing algorithms on the digitized sound signal to increase the comprehensibility [3]. Even though these can perform diverse functions such as feedback

Tomson Devis

tomsondevis@gmail.com

Manju Manuel

manjum@rit.ac.in

1 APJ Abdul Kalam Technological University, Thiruvananthapuram, Kerala, India

2 Department of Electronics and Communication Engineering, Rajiv Gandhi Institute of Technology, Kottayam, Kerala 686501, India cancellation, speech enhancement and noise reduction, auditory compensation is its principal function [4]. In addition to the quality of sound signal decomposition and amplification, the degree of hearing loss also influences the effectiveness in auditory compensation [5].

The most common type of hearing loss is Sensorineural Hearing Loss (SNHL). In this type of hearing loss, the sound sensing cells in the inner ear are damaged and has the possibility of occurrence at any age. Recently, it has been identified that coronavirus (SARS-CoV-2) can also be one of the reasons for sudden SNHL [6]. Another type of hearing loss commonly affecting the elderly, which results in a simultaneous reduction in the functioning of both ears is Presbycusis [7]. Majority of the hearing assistive devices available today are used to compensate SNHL. The hearing loss characteristics of the impaired person are corrected using hearing aids with the help of various auditory compensation techniques. These techniques are used for adjusting the magnitude response of the filters in an inverted fashion of the hearing deficiency, in such a way that, more gain is applied to the filter in the position of deep hearing 
deficiency. Unfortunately, the global production of hearing aids is able to meet only less than $10 \%$ of the requirement $[8,9]$. Thus, efficient hearing aids with reduced complexity both in design and hardware has to be evolved for addressing this severe shortage of hearing aids.

Hearing aid users enjoy the proven clinical benefits of improved communication and reduced listening effort. The use of hearing aids provide better connectivity for them with the surrounding society which boosts their confidence and well-being to a large extent. A properly adjusted hearing aid can improve the speech, language, and social skills of hearing deprived children [10]. The use of hearing aid can postpone the onset of reduced cognitive functioning and development of dementia which might otherwise lead to Alzheimer's disease [11]. An untreated hearing loss can stimulate atrophy in the auditory regions of the brain in older adults, making speech comprehension difficult in future [12]. Thus, the timely use of hearing aids can help in reducing atrophy while increasing the hearing ability and may also slow down the cognitive decline associated with hearing loss.

Hearing defects are minimized effectively by providing selective gain on each band of the audio spectrum by utilizing the filter bank available in digital hearing aids. Initially, the sub-bands were separated using uniform filter banks which provided only moderate correction for hearing impairments. Later, non-uniform filter banks were employed for sub-band generation to get better compensation for hearing impairments. Several techniques have been proposed to date for the generation of sub-bands using non-uniform filter banks. One of the common techniques employed for this purpose uses an ANSI S1.11 filter bank. In this method, all the octaves from $250 \mathrm{~Hz}$ to $8 \mathrm{kHz}$ are covered and the most efficient matching is obtained [13]. But this method resulted in wastage of hardware along with substantial device complexity and group delay when compared to other methods. Even though a Quasi-ANSI S1.11 filter bank method is developed with reduced processing delay, its level of complexity is high [14]. Frequency Response Masking (FRM) is yet another method for generating non-uniform filter banks $[15,16]$. In this technique, low complexity wideband filters are used to create narrowband filters with a sharp transition, but have more delay than other techniques $[17,18]$. Transition filter and adjacent band merging approaches are applied on Cosine Modulated (CM) prototype filter for generating non-uniform filter banks but have the disadvantage of increased complexity [19].

The Variable Bandwidth Filter (VBW) employs a sampling rate conversion technique for making non-uniform sub-bands [20]. But complex hardware is required for the realization of this reconfigurable technique. Even though a modified VBW method using farrow structure was successful in reducing the delay with better matching results, the device complexity increased further [21, 22]. The modified Discrete Fourier Transform (MDFT) method proposed for generating non-uniform filter banks also required very complex hardware for implementation [23]. Fractional Interpolation (FI) method used for the creation of non-uniform filter banks has moderate complexity [24]. But this method produces considerable delay than the permitted delay of 20 ms while adjusting audiograms having acute variations in hearing level. A modification of this FI method with a twolevel sound wave decomposition is also developed in [25]. A 17-band fixed filter bank structure which uses the interpolation of the prototype filter is also developed, but with moderate complexity level [26]. Thus, even though several techniques have been developed for the generation of filter banks, most of these methods are of high complexity which demands complex hardware elements thereby increasing the cost. Also, existing reconfigurable techniques employ manual interventions during the design stage to adjust proper bands in the respective frequency positions which is a tiresome and time-consuming task [19, 23, 24]. In this paper, a low complexity, auto-reconfigurable hearing aid which can adapt to optimum bands by itself in various positions is proposed.

\section{Finite impulse response filters}

Digital filters can be realized using Finite Impulse Response (FIR) method and Infinite Impulse Response (IIR) method. The non-recursive nature of the FIR filter makes them best suited for audio processing applications. The impulse response of the FIR filter is limited to a finite interval. FIR filter has a linear phase response which is crucial for auditory compensation applications in hearing aids. The inherent property of linear phase FIR filters to preserve phase information in the incoming audio signal is essential for sound localization and echo cancellation procedures in digital hearing aids [27]. Besides, the FIR filters have greater stability and more flexible implementation capabilities than IIR filters. No phase distortion is observed in FIR filters, which is essential in audio processing applications [28]. The difference equation of $N$ th order FIR filter is given in Eq. (1). The output sequence $y(n)$ is the convolution sum of the input sequence $x(n)$ and the impulse response $h(n)$.

$y(n)=\sum_{k=0}^{N} h(k) x(n-k)$

The transfer function of the FIR filter of order $N$ is given by

$H(z)=\sum_{n=0}^{N} h(n) z^{-n}$ 
The symmetric coefficients of the linear phase FIR filter are represented by

$h(n)=h(N-n)$ for $n=0,1,2, \ldots, N$

A linear phase FIR filter is realized effectively using the Remez Exchange algorithm or Parks-McClellan algorithm [29]. An optimized least order FIR filter having equi-ripple passband and stopband is generated with the given specifications. The Kaiser formula based Parks-McClellan algorithm for the design of an FIR filter of order $N$ is given by

$N=\frac{-20 \log _{10}\left(\sqrt{\delta_{p} \delta_{s}}\right)-13}{14.6\left(\omega_{s}-\omega_{p}\right) / 2 \pi}$

where the maximum allowed ripples in the passband and stopband are $\delta_{p}$ and $\delta_{s}$, and the passband and stopband edge frequencies are $\omega_{p}$ and $\omega_{s}$ respectively. This least order FIR filter design method demands minimum number of adders and multipliers. $N$ adders are required for this optimized design and the number of multipliers needed is given by

$\# m u l t=\left\lceil\frac{N+1}{2}\right\rceil$

\section{Octave and fractional interpolated filters}

Multirate signal processing methods are employed in digital systems where sampling rate conversions are required. In multirate signal processing, the passband width of the prototype filter is varied by the interpolation and decimation techniques [30]. An interpolation by $L$ operation will increase the sampling rate by a factor $L$ in time domain and will provide $L$-fold bandwidth compression of the passband in frequency domain. Similarly, the $D$-fold decimation operation decrease the sampling rate by a factor $D$ in time domain and expand the passband $D$ times in frequency domain [31]. The time-domain representation of the interpolation is the insertion of $L-1$ null samples between each original sample. Likewise, the decimation is achieved by selecting every Dth sample of the original sequence. The interpolation and decimation operations are represented in the frequency domain as $H\left(z^{L}\right)$ and $H\left(z^{1 / D}\right)$ respectively.

In octave interpolation, the interpolation factor $L$ is a dyadic function of $K$, which is given as $L=2^{K}$. The different levels of octave interpolation are represented by $K=1,2, \ldots, M$. The $M t h$ level octave interpolation is obtained when $K=M$. In octave interpolation of the prototype filter, the number of passband is increased dyadically, which will perfectly fit the particular region. The fractional interpolation is a combination of the interpolation and decimation operations and described as $H\left(z^{L / D}\right)$. In $H\left(z^{L / D}\right)$, the interpolation and decimation factors, $L$ and $D$ are relatively prime. In $H\left(z^{L / D}\right)$, a group of every Dth coefficients of the prototype filter are taken and $(L-1)$ null samples are inserted in between the selected coefficients [32].

The remaining sections of this paper are organized as follows: Section 2 gives an overview of the methodology of the proposed reconfigurable filter bank structure. The evaluation results are given in section 3 . The experimental results are discussed in section 4 and finally, the conclusions are given in section 5 .

\section{Methods}

The prototype filter, $H_{l}(z)$ is designed using the ParksMcClellan algorithm with a bandwidth of $\pi / 3$. The symmetric coefficients of the linear phase FIR filter $H_{l}(z)$ are used for further interpolation and decimation operations. In the proposed method, the overall frequency response of the human ear is divided equally into three regions. The division of the hearing spectrum in this manner makes it very easy to interpret the hearing deficiencies of the patient in the low, mid, and high frequencies by an audiologist or a hearing expert. The magnitude response of $H_{l}(z)$ itself constitutes the first region of the hearing spectrum, which handles the audio frequencies up to $2.67 \mathrm{kHz}$. A high pass filter $H_{h}(z)$ is created from $H_{l}(z)$ using frequency transformation. $H_{h}(z)$ deals with the frequencies ranging from 5.34 to $8 \mathrm{kHz}$ of the hearing spectrum, which represents the third region. A middle frequency bandpass filter, $H_{m}(z)$ is produced from $H_{l}(z)$ and $H_{h}(z)$ using spectrum subtraction. This filter handles the frequencies ranging from 2.67 to $5.34 \mathrm{kHz}$ in the second region of the hearing spectrum. The equations used for generating the sub-filters from the prototype filter with various interpolation and decimation factors, $L$ and $D$ respectively are listed in Table 1. The parameter ' $\Delta$ ' in the equations represent the half-length of the corresponding filter.

Table 1 Equations used for generating octave and fractional interpolated filters

\begin{tabular}{lll}
\hline $\begin{array}{l}\text { Interpolation fac- } \\
\text { tor, } \mathrm{L}\end{array}$ & $\begin{array}{l}\text { Decimation fac- } \\
\text { tor, D }\end{array}$ & Filter representations \\
\hline 1 & 1 & $H_{h}(z)=z^{-\Delta}-H_{l}(z)$ \\
1 & 1 & $H_{m}(z)=1-H_{l}(z)-H_{h}(z)$ \\
2 & 1 & $H_{h}\left(z^{2}\right)=z^{-\Delta}-H_{l}\left(z^{2}\right)$ \\
2 & 1 & $H_{m}\left(z^{2}\right)=1-H_{l}\left(z^{2}\right)-H_{h}\left(z^{2}\right)$ \\
4 & 1 & $H_{h}\left(z^{4}\right)=z^{-\Delta}-H_{l}\left(z^{4}\right)$ \\
4 & 1 & $H_{m}\left(z^{4}\right)=1-H_{l}\left(z^{4}\right)-H_{h}\left(z^{4}\right)$ \\
8 & 1 & $H_{h}\left(z^{8}\right)=z^{-\Delta}-H_{l}\left(z^{8}\right)$ \\
8 & 1 & $H_{m}\left(z^{8}\right)=1-H_{l}\left(z^{8}\right)-H_{h}\left(z^{8}\right)$ \\
2 & 3 & $H c\left(z^{2 / 3}\right)=z^{-\Delta}-H_{l}\left(z^{2 / 3}\right)$ \\
4 & 3 & $H c\left(z^{4 / 3}\right)=z^{-\Delta}-H_{l}\left(z^{4 / 3}\right)$ \\
\hline
\end{tabular}


In the proposed design, four different schemes are suggested in each region for sub-band decomposition. The number of bands assigned in each region and the corresponding bandwidth are given in Table 2. Since the scheme 1 uses only one band in each region, the base filters $H_{l}(z), H_{m}(z)$

Table 2 Number of bands and bandwidths in different schemes

\begin{tabular}{lllll}
\hline Scheme & \multicolumn{2}{l}{ Number of bands } & Bandwidth \\
\cline { 2 - 4 } & Region 1 & Region 2 & Region 3 & \\
\hline Scheme 1 & 1 & 1 & 1 & $\pi / 3$ \\
Scheme 2 & 2 & 2 & 2 & $\pi / 6$ \\
Scheme 3 & 3 & 3 & 3 & $\pi / 6, \pi / 12$ \\
Scheme 4 & 6 & 5 & 6 & $\pi / 12, \pi / 24$ \\
\hline
\end{tabular}
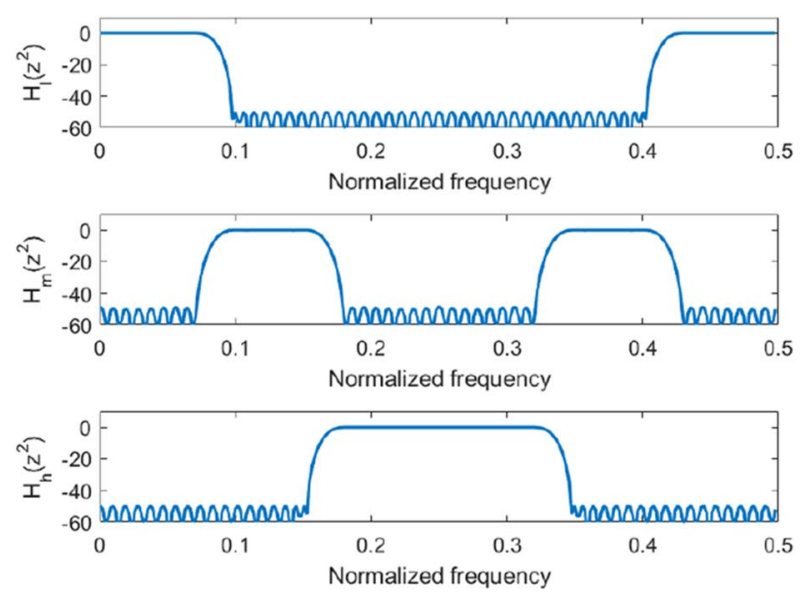

(a) Magnitude responses of the level-1 octave interpolated filters
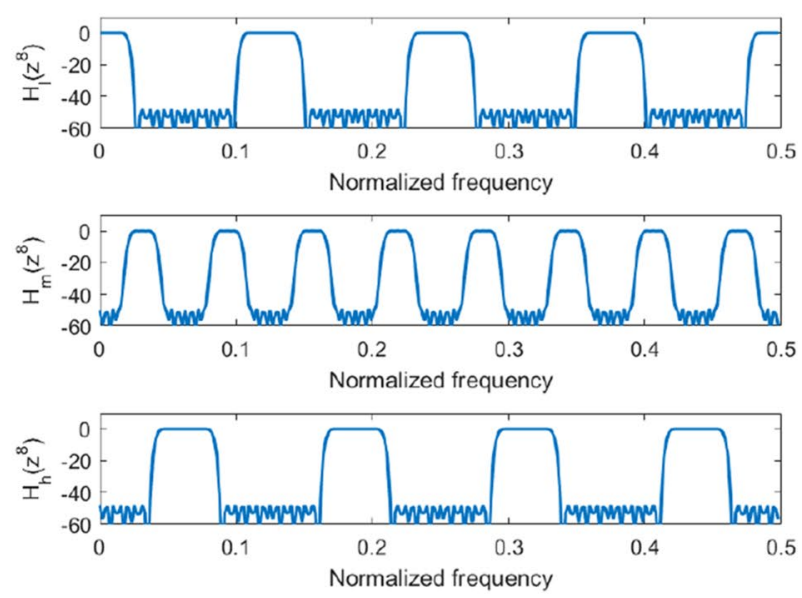

(c) Magnitude responses of the level-3 octave(d) interpolated filters and $H_{h}(z)$ itself are assigned as the sub-bands of scheme 1 . These filters are also used as masking filters for selecting the particular region when higher schemes are used. Three-level octave interpolations are carried on $H_{l}(z), H_{m}(z)$ and $H_{h}(z)$ to generate more number of sub-bands with lesser bandwidths. The corresponding interpolated filters are represented as $H_{l}\left(z^{L}\right), H_{m}\left(z^{L}\right)$ and $H_{h}\left(z^{L}\right)$. These sub-filters are cascaded with the masking filters of each region to get the sub-bands in that particular region. In level-1 octave interpolation, $K=1$ is selected for generating the sub-bands of scheme 2 . The resultant filters are symbolized as $H_{l}\left(z^{2}\right), H_{m}\left(z^{2}\right)$ and $H_{h}\left(z^{2}\right)$ and the magnitude responses are shown in Fig. 1a. A 6-band uniform filter bank with a bandwidth of $\pi / 6$ is obtained from these filters and the filter bank have two bands in each region.
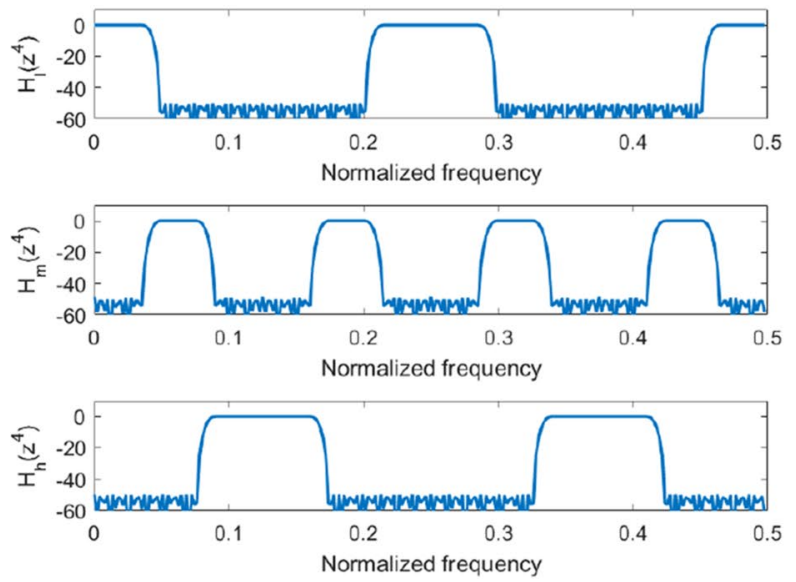

(b) Magnitude responses of the level-2 octave interpolated filters
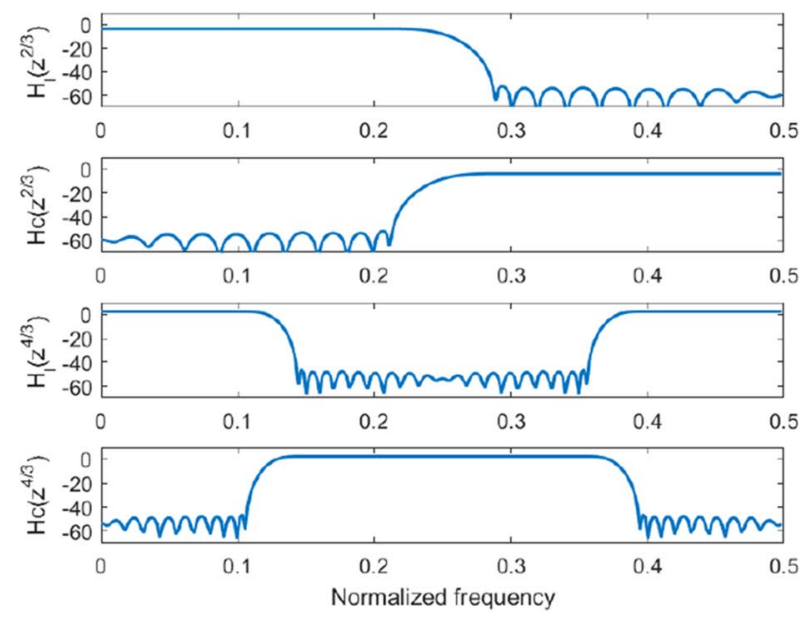

(d) Magnitude responses of the fractional interpolated filters

Fig. 1 Magnitude responses of octave and fractional interpolated filters 
In level-2 octave interpolation, $K=2$ is selected for generating the sub-bands of scheme 3 . The resultant filters are symbolized as $H_{l}\left(z^{4}\right), H_{m}\left(z^{4}\right)$ and $H_{h}\left(z^{4}\right)$ and the magnitude responses are shown in Fig.1b. A 9-band non-uniform filter bank is synthesized from these sub-filters, and three sub-bands are present in each region. The bandwidth of the broader band is $\pi / 6$, and that of the narrower band is $\pi / 12$. The scheme 4 sub-bands are obtained by selecting $K=3$ and the corresponding level-3 octave interpolated filters are represented as $H_{l}\left(z^{8}\right), H_{m}\left(z^{8}\right)$ and $H_{h}\left(z^{8}\right)$. The magnitude responses of these interpolated filters are illustrated in Fig. 1c. A 17-band non-uniform filter bank is generated from these filters in which, six bands are present in the first and third regions, and five bands in the second region. The bandwidth of the broader band is $\pi / 12$ and that of the narrower band is $\pi / 24$.

Some of the sub-bands use common cascaded filters in scheme 2, 3 and 4 . These sub-bands are separated using fractional interpolated filters $H_{l}\left(z^{2 / 3}\right)$ and $H_{l}\left(z^{4 / 3}\right)$ as well as its complementary pairs $H c\left(z^{2 / 3}\right)$ and $H c\left(z^{4 / 3}\right)$. The magnitude responses of these filters are illustrated in Fig. 1d. The set of equations for generating the fractional interpolated and the complementary filters are listed in Table 1 . The magnitude responses of the bands of different schemes in each region are illustrated in Fig. 2. Figure $2 \mathrm{a}-\mathrm{c}$ are the sub-bands of scheme 1 which have only one band in each region. Figure $2 \mathrm{~d}-\mathrm{f}$ are the sub-bands of scheme 2 with two bands in different regions. Similarly, Fig. $2 \mathrm{~g}-\mathrm{i}$ are the sub-bands of scheme 3 with three bands in each region. Further, Fig. $2 j-1$ are the sub-bands of scheme 4 . In scheme 4 , there are six bands in the first and third regions as well as five bands in the second region. The sub-bands are denoted as $S_{i j k}$, where $i, j$, and $k$ are the region, scheme, and the band position respectively. For example, the sub-band named ' $S_{231}$ ' defines the first band in the second region, when scheme 3 is used.

\section{Selection of optimum scheme for auto-reconfigurability}

Auto-reconfigurability in the hearing aid is accomplished by selecting the optimum scheme in each region. The schemes proposed in each region depends on the severity of hearing loss in the particular region. Lower schemes are used in a region when response of the impaired ear is almost flat in that region. Whenever there is a sharp variation in any of the regions in the audiogram, higher schemes are preferred in that region. An audiogram describes the mildest sound that can be heard at different test frequencies of $250 \mathrm{~Hz}, 500 \mathrm{~Hz}$, $1 \mathrm{kHz}, 2 \mathrm{kHz}, 4 \mathrm{kHz}$, and $8 \mathrm{kHz}$ by the hearing impaired [33]. The audiogram has six distinct hearing threshold values which are represented as $a_{i}$ at different octaves. The gradients between adjacent octaves are calculated as $g_{i}=a_{i+1}-a_{i}$, and the maximum gradient in any region is considered for the optimum scheme selection. Since there are six octaves in an audiogram, five gradients are present in the audio spectrum, which are represented as $g_{i}$. The first three gradients and the (1/3)rd of the fourth gradient, $g_{4}$ are located in the first region. The remaining $(2 / 3) r d$ portion of $g_{4}$ along with $(1 / 3) r d$ of $g_{5}$ are in region 2 . The remaining $(2 / 3) r d$ portion of $g_{5}$ belongs to region 3 .

The rising or falling trend of the hearing deficiency in any region is estimated by the compounding of gradients in the corresponding region. The maximum value of the gradients in any region is considered for the estimation of optimum scheme in the particular region. The maximum value of gradients in the $j^{\text {th }}$ region is termed as the slope value, $D_{j}$ as listed in Table 3 . The optimum scheme in any region is deduced from the slope values. If $D_{j}$ is within the upper threshold of $5 \mathrm{~dB}$, scheme 1 is suggested in the region, $j$. Similarly, when $D_{j}$ is within the upper threshold of $10 \mathrm{~dB}$, scheme 2 is suggested in the corresponding region. Again, an upper threshold of $15 \mathrm{~dB}$ suggests scheme 3 and that of $30 \mathrm{~dB}$ suggests scheme 4 in the respective regions.

\section{Structure of the proposed method}

The structure of the proposed reconfigurable filter bank is shown in Fig. 3. Each filter have two outputs as represented by symbols 'o' and ' $c$ ', which are the output of the original and the complementary filter respectively. The sub-bands of different schemes are selected using a 3-bit control switch, $S_{1} S_{2} S_{3}$. This switch will enable the different stages of the proposed structure. A bit ' 1 ' in the control signal will close the normally opened switches. Initially, the sub-bands for scheme 1 are generated using the filter $H_{l}(z)$, and the results are stored in storage 1 , which also defines the three regions. The switch status ' 100 ' produces the sub-bands of scheme 2 and the results are stored in storage 2 . This switch status will enable the filters $H_{l}(z), H_{l}\left(z^{2}\right), H_{l}\left(z^{2 / 3}\right)$ and storage 2 . The switch status ' 110' produces the sub-bands of scheme 3 by enabling the filters $H_{l}(z), H_{l}\left(z^{2}\right), H_{l}\left(z^{4}\right), H_{l}\left(z^{2 / 3}\right)$ and storage 3 for storing the results. Similarly, the switch status ' 111 ' produces the sub-bands of scheme 4 by enabling the filters $H_{l}(z), H_{l}\left(z^{2}\right), H_{l}\left(z^{4}\right), H_{l}\left(z^{8}\right), H_{l}\left(z^{2 / 3}\right), H_{l}\left(z^{4 / 3}\right)$ and storage 4 which stores the results.

\section{Results}

\section{Optimal transition width of the prototype filter}

The prototype filter, $H_{l}(z)$ is designed using the ParksMcClellan equi-ripple algorithm with the following specifications. The sampling frequency, $f_{s}$ is chosen as $16 \mathrm{kHz}$, ripples in the passband, $\delta_{p}$ is limited to $0.05 \mathrm{~dB}$, and the stopband attenuation is selected as $50 \mathrm{~dB}$. The transition 


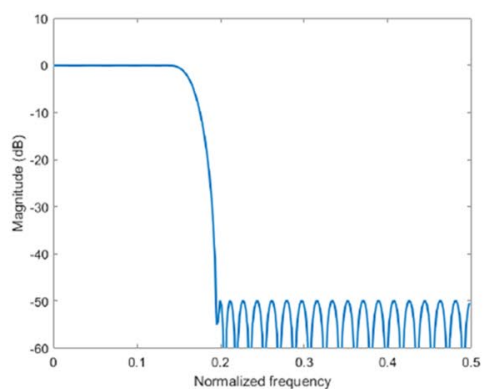

(a) $S_{111}$

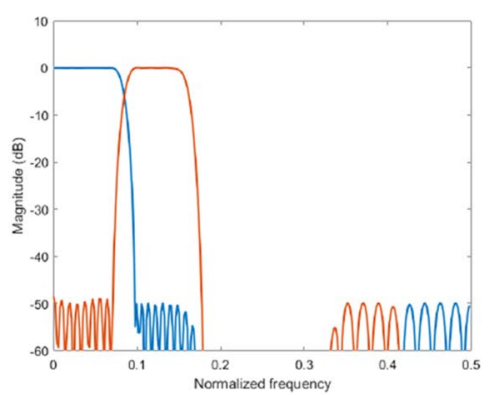

(d) $S_{121}, S_{122}$

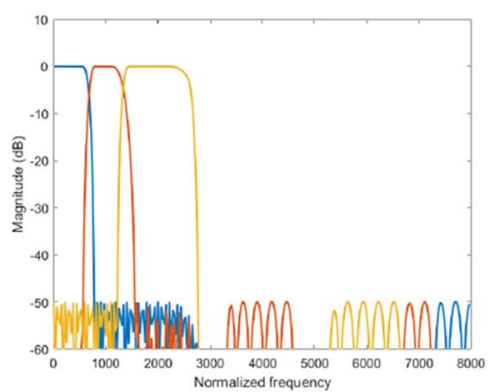

(g) $S_{131}, S_{132}, S_{133}$

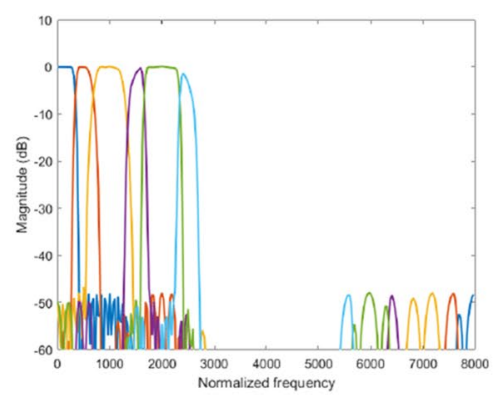

(j) $S_{141}, S_{142}, S_{143}, S_{144}, S_{145}, S_{1}$ (1) $S_{241}, S_{242}, S_{243}, S_{244}, S_{245}$
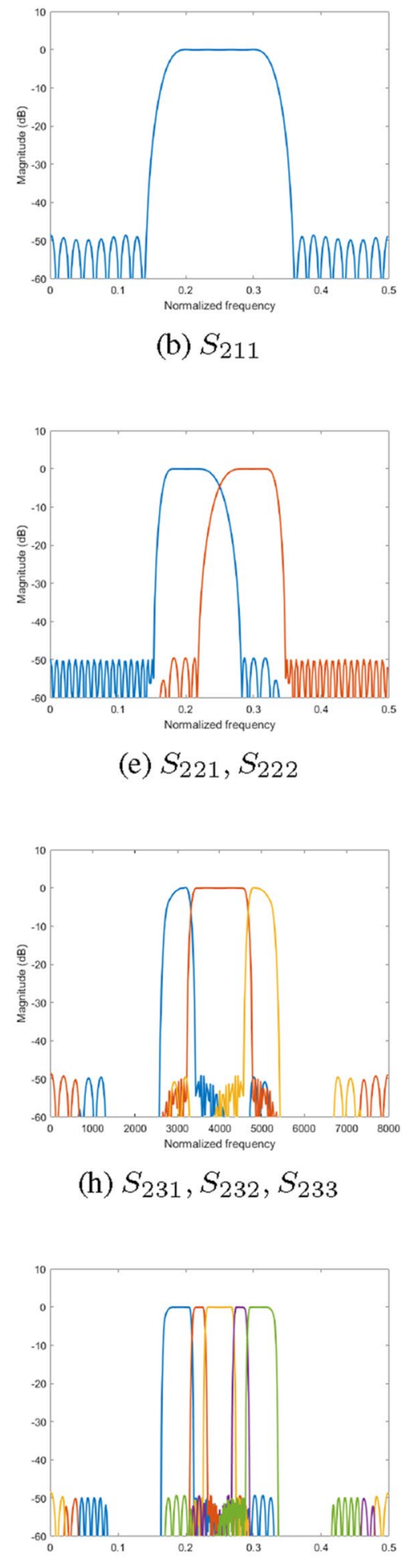

(b) $S_{211}$

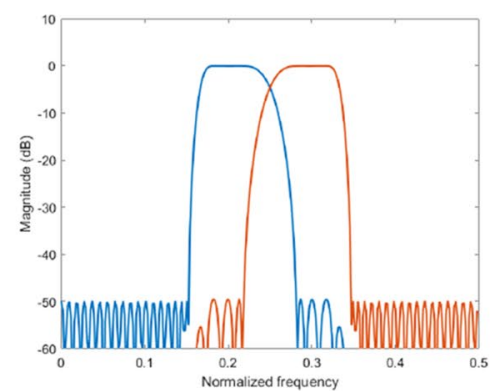

(e) $S_{221}, S_{222}$

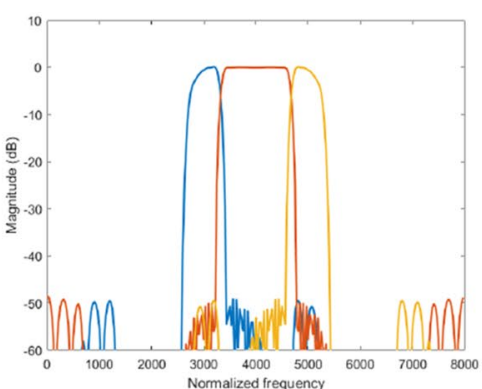

(h) $S_{231}, S_{232}, S_{233}$ (i) $S_{331}, S_{332}, S_{333}$
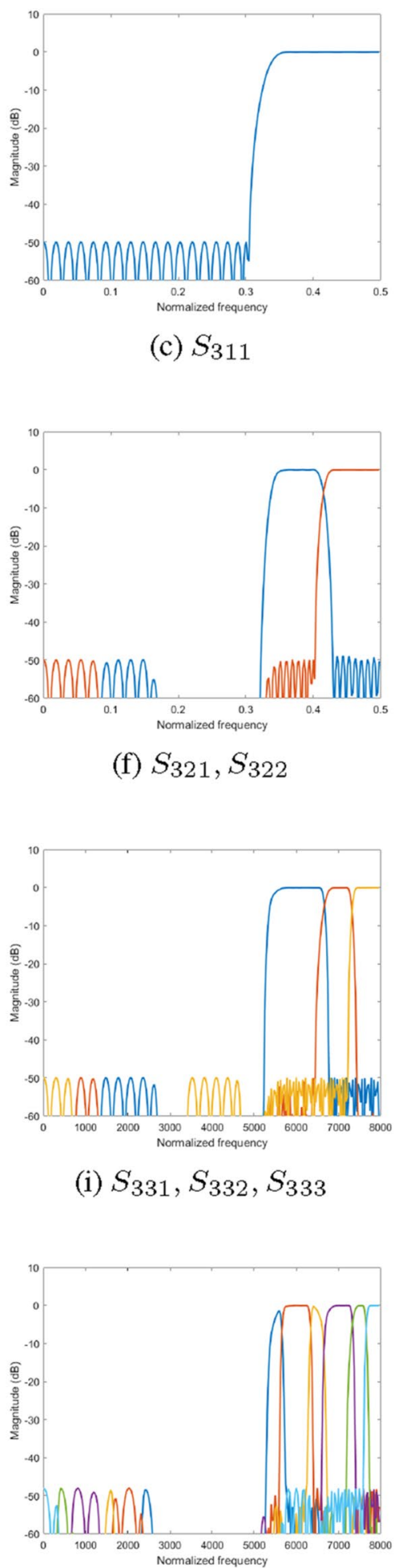

(c) $S_{311}$

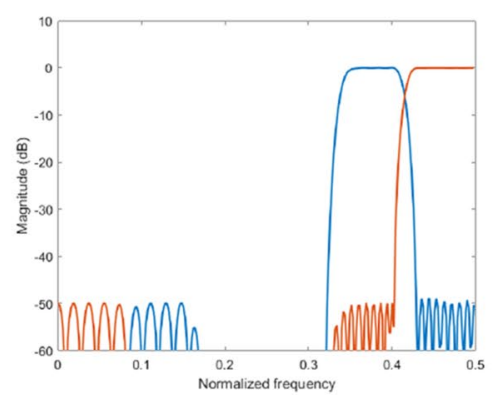

(f) $S_{321}, S_{322}$

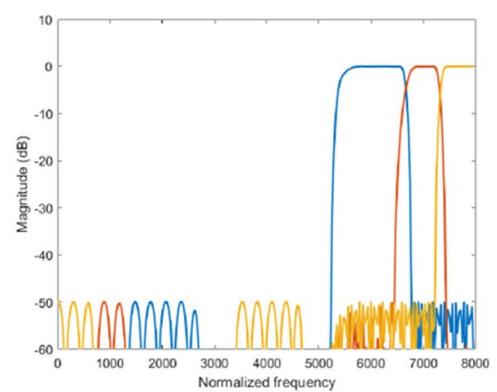

(1) $S_{341}, S_{342}, S_{343}, S_{344}, S_{345}, S_{346}$

Fig. 2 Sub-bands of scheme 1, scheme 2, scheme 3, and scheme 4 in different regions

width of the prototype filter determines the complexity of the entire system. Besides, the transition width also defines the sharpness of the sub-filters used in higher schemes. The estimation of optimum transition width is done with the matching of the right ear of audiogram 1 in Fig. 4, which has mild hearing loss in all frequencies. 
Table 3 Slope values in different regions

\begin{tabular}{lll}
\hline Region & Frequency range & Slope values \\
\hline 1 & $0-2.67 \mathrm{kHz}$ & $D_{1}=\max \left(\left|g_{1}\right|,\left|g_{2}\right|,\left|g_{3}\right|,\left|g_{4} / 3\right|\right)$ \\
2 & $2.67 \mathrm{kHz}-5.34 \mathrm{kHz}$ & $D_{2}=\max \left(\left|2 g_{4} / 3\right|,\left|g_{5} / 3\right|\right)$ \\
3 & $5.34 \mathrm{kHz}-8 \mathrm{kHz}$ & $D_{3}=\left|2 g_{5} / 3\right|$ \\
\hline
\end{tabular}

Various designs of the prototype filter with normalized transition width between 0.1 and 0.2 are examined. The corresponding filter order and the maximum matching error (MME) in the auditory compensation procedure are listed in Table 4. It is evident from the table that the least matching error is obtained at a transition width of 0.175 . Further increment in transition width will deliver higher errors due to the increased overlapping of adjacent bands. Hence, the best selection of the transition width and the order of the prototype filter, $H_{l}(z)$ in the proposed system are 0.175 and 35 respectively. According to Eq. (5), the effective implementation of the prototype filter can be carried out with a minimal number of 18 multipliers.

\section{Delay analysis of the proposed system}

In addition to the use of hearing assistive devices, the hearing-impaired people closely observe the lip movements of the speaker to improve their hearing perception. To ensure good synchronization, the maximum processing delay of the hearing device is limited to $20 \mathrm{~ms}[34]$. Else, it will cause a mismatch in the synchronization between the visual lipreading and the processed audio output of the device. The group delay associated with an FIR filter having different levels of interpolation and decimation is given by
Fig. 3 Structure of the proposed system
Table 4 Selection of optimal transition width of the prototype filter

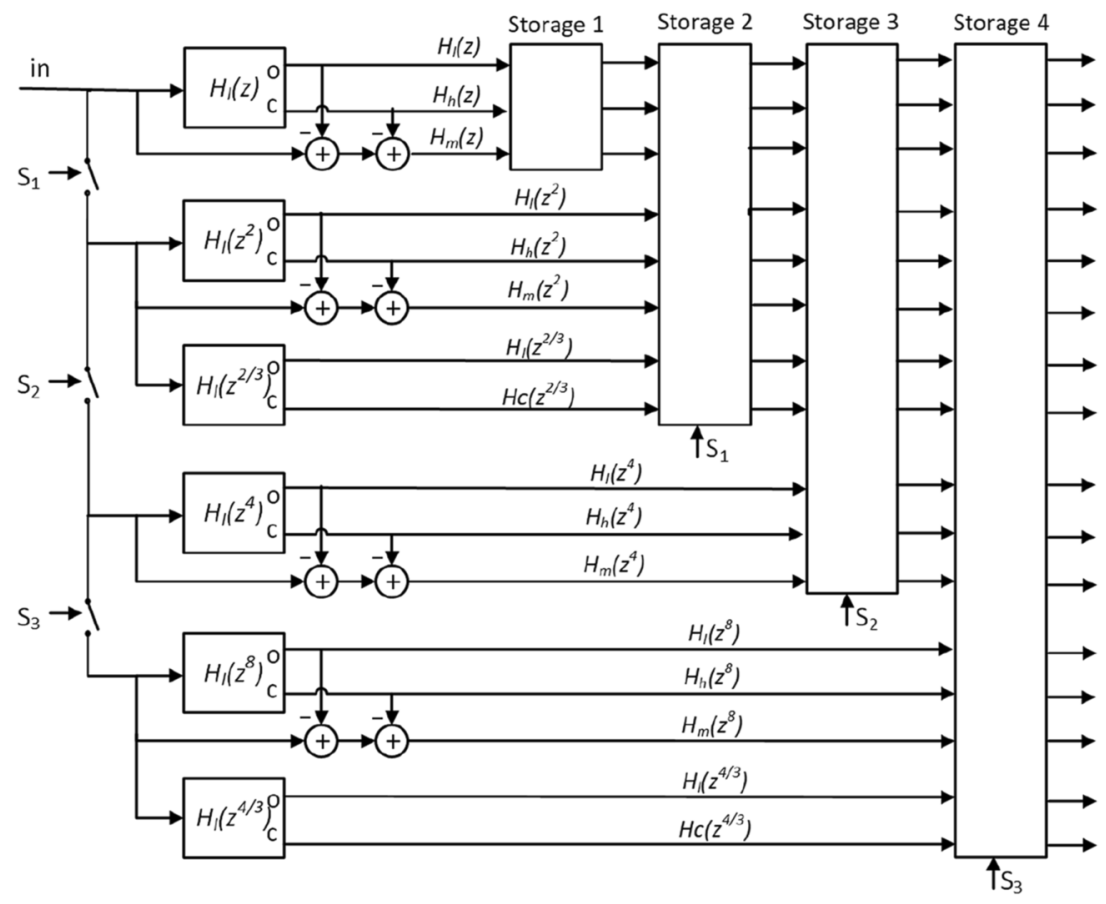

\begin{tabular}{|c|c|c|c|c|c|}
\hline Transition width & $\begin{array}{l}\text { Passband edge, } \\
w_{p}\end{array}$ & $\begin{array}{l}\text { Stopband edge, } \\
w_{s}\end{array}$ & Filter order & $\begin{array}{l}\text { Multipliers } \\
\text { required }\end{array}$ & $\operatorname{MME}(\mathrm{dB})$ \\
\hline 0.1000 & .2834 & .3834 & 62 & 31 & 4.08 \\
\hline 0.1125 & .2771 & .3896 & 55 & 28 & 3.82 \\
\hline 0.1250 & .2709 & .3959 & 49 & 25 & 3.51 \\
\hline 0.1375 & .2646 & .4021 & 45 & 23 & 3.13 \\
\hline 0.1500 & .2584 & .4084 & 41 & 21 & 2.78 \\
\hline 0.1625 & .2521 & .4146 & 38 & 19 & 2.47 \\
\hline 0.1750 & .2459 & .4209 & 35 & 18 & 2.28 \\
\hline 0.1875 & .2396 & .4271 & 33 & 17 & 2.36 \\
\hline 0.2000 & .2334 & .4334 & 30 & 16 & 2.49 \\
\hline
\end{tabular}


$T=\frac{N L}{2 D f_{s}}$

where $N$ represents the order of the prototype filter $H_{l}(z), f_{s}$ is the sampling rate of the system, $L$ and $D$ are the interpolation and the decimation factors respectively.

According to Eq. (6), the processing delay of the bands in scheme 1 , scheme 2 , scheme 3 , and scheme 4 are $1.09 \mathrm{ms,}$ $4 \mathrm{~ms}, 8.36 \mathrm{~ms}$, and $18.54 \mathrm{~ms}$ respectively. The delay of the proposed method is compared with existing audibility restoration techniques and is given in Table 5. The number of bands in the fixed filter bank method is constant whereas the bands of the reconfigurable filter bank method depends on the selected scheme. The proposed auto-reconfigurable filter bank structure has three distinct regions and four different schemes are present in each region. Hence, the number of bands in the structure can vary from 3 to 17 . The maximum delay of the system is within the acceptable limit of $20 \mathrm{~ms}$, even when the scheme 4 is used in all regions.

\section{Verification of audiogram matching capability}

The audiogram is a graphical representation of the performance of human ear at different frequencies, which is estimated by Pure Tone Audiometry (PTA). In PTA, the responses of the ear are measured by applying each octave frequencies between $250 \mathrm{~Hz}$ and $8 \mathrm{kHz}$. The responses of the ear are represented in decibels $(\mathrm{dB})$ in $\mathrm{Y}$-axis, and the test frequencies in hertz $(\mathrm{Hz})$ are represented in $\mathrm{X}$-axis. In the audiogram, a symbol ' $\mathrm{O}$ ' is used for representing the response of right ear, and a symbol ' $\mathrm{X}$ ' is used for that of the left ear. The hearing thresholds until $20 \mathrm{~dB}$ are regarded as normal hearing and the values between 20 to $40 \mathrm{~dB}$ represent a mild hearing loss. The range of values between 40 to $70 \mathrm{~dB}$ indicates a moderate hearing loss and 70-90dB are the signs of severe hearing loss. The hearing thresholds beyond $90 \mathrm{~dB}$ shows a profound hearing loss [35].

Different categories of audiograms having various hearing impairments as shown in Fig. 4 are effectively compensated with the proposed auto-reconfigurable system. The test audiograms shown in Fig. 4 are collected from the audiometry screening process [36] and the independent hearing aid information, a public service provider of Hearing Allianz of America [37]. In PTA, an air conduction test is performed on both ears of the impaired and it is observed that most of the audiograms show a bilateral hearing loss. An almost alike failure occurs in both ears in the case of bilateral hearing loss; otherwise, it is called unilateral hearing loss. The audiogram matching of the proposed method is compared with three existing techniques and the results are listed in Table 6 . The proposed reconfigurable filter bank structure ensures better results by effectively compensating almost all audiograms within the acceptable limit of $\pm 3 \mathrm{~dB}$ [22].

In all the existing reconfigurable hearing aid systems, manual interventions are applied for the best scheme selection [19, 23, 24]. The proposed reconfigurable system can automatically select the best-suited scheme in each region for all audiograms. In the proposed optimum scheme selection method, the variations in hearing deficiency at different octaves are evaluated and used for choosing the best scheme. The frequency responses of the matched filter banks and the corresponding matching results are shown in Fig. 5. The optimum scheme which is assigned in each region for the test audiograms by the proposed auto-reconfigurable method and the corresponding maximum group delays of the selected schemes are listed in Table 7. Even the audiogram having sharp variations in hearing profile in all regions can be successfully matched within the permitted delay of $20 \mathrm{~ms}$.

\section{Discussion}

\section{Reduction in complexity}

Parks-McClellan equi-ripple algorithm is utilized for the design of prototype filter. The proposed reconfigurable filter bank structure has very meagre complexity than other filter bank generation methods in the literature. Since the coefficient multiplier is the most resource-hungry component in the hardware, the complexity of the proposed structure is estimated by the number of coefficient multipliers used
Table 5 Comparison of group delay of the proposed system with existing methods

\begin{tabular}{llll}
\hline Method & Type of filter bank & Number of bands & $\begin{array}{l}\text { Maximum } \\
\text { delay (ms) }\end{array}$ \\
\hline ANSI S1.11 Filter bank[13] & Fixed & 18 & 31 \\
Quasi-ANSI S1.11 Filter bank[14] & Fixed & 18 & 10 \\
Frequency Response Masking [16] & Fixed & 8 & 26.6 \\
Variable Bandwidth Filters [22] & Reconfigurable & 4 to 10 & 1.1 \\
Fractional Interpolation (FI) [24] & Reconfigurable & 3 to 12 & 21.6 \\
Two-level FI [25] & Reconfigurable & 3 to 13 & 18.5 \\
Proposed method & Reconfigurable & 3 to 17 & 18.54 \\
\hline
\end{tabular}




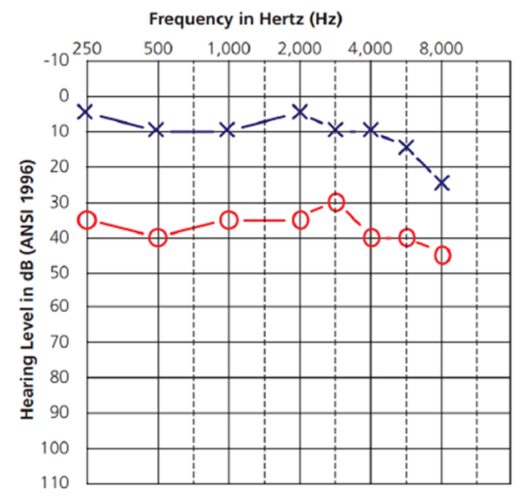

(a) Audiogram 1

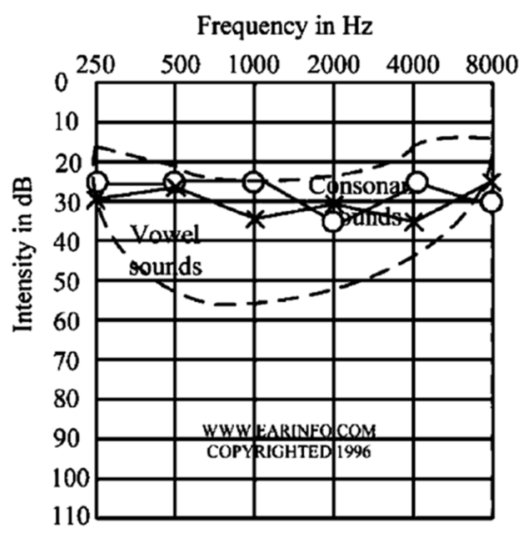

(b) Audiogram 2

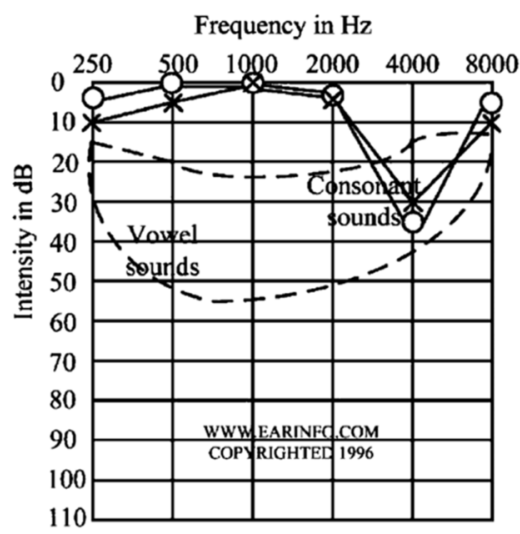

(c) Audiogram 3

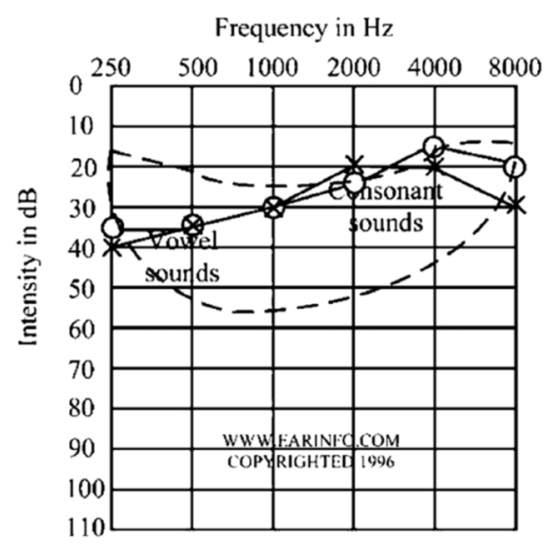

(d) Audiogram 4

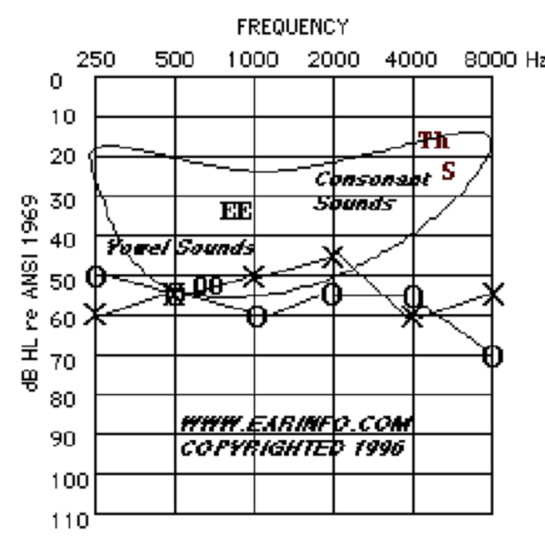

(e) Audiogram 5

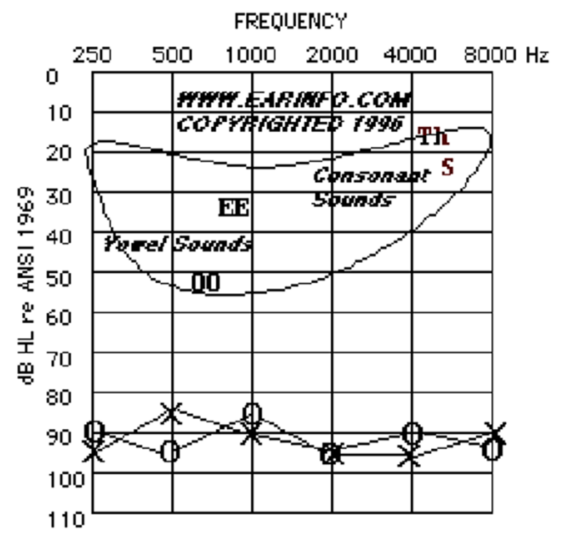

(f) Audiogram 6

Fig. 4 Test audiograms used for evaluation purposes [36, 37]

Table 6 Comparison of audiogram matching errors

\begin{tabular}{|c|c|c|c|c|c|}
\hline Test audiogram & Type of hearing loss (HL) & Method in [24] & Method in [26] & Method in [25] & Proposed method \\
\hline Audiogram 1 left ear & Mild HL in high freq & $3.13 \mathrm{~dB}$ & $2.98 \mathrm{~dB}$ & $2.69 \mathrm{~dB}$ & $2.61 \mathrm{~dB}$ \\
\hline Audiogram 1 right ear & Mild HL in all freq & $2.83 \mathrm{~dB}$ & $2.67 \mathrm{~dB}$ & $2.32 \mathrm{~dB}$ & $2.28 \mathrm{~dB}$ \\
\hline Audiogram 2 left ear & Mild HL in all freq & $2.01 \mathrm{~dB}$ & $2.14 \mathrm{~dB}$ & $2.71 \mathrm{~dB}$ & $1.89 \mathrm{~dB}$ \\
\hline Audiogram 2 right ear & Mild HL in all freq & $1.84 \mathrm{~dB}$ & $3.17 \mathrm{~dB}$ & $2.18 \mathrm{~dB}$ & $1.91 \mathrm{~dB}$ \\
\hline Audiogram 3 left ear & Mild HL in high freq & $5.27 \mathrm{~dB}$ & $3.76 \mathrm{~dB}$ & $2.77 \mathrm{~dB}$ & $2.90 \mathrm{~dB}$ \\
\hline Audiogram 3 right ear & Mild HL in high freq & $5.63 \mathrm{~dB}$ & $3.82 \mathrm{~dB}$ & $2.92 \mathrm{~dB}$ & $2.83 \mathrm{~dB}$ \\
\hline Audiogram 4 left ear & Mild HL in all freq & $3.12 \mathrm{~dB}$ & $2.90 \mathrm{~dB}$ & $2.86 \mathrm{~dB}$ & $2.41 \mathrm{~dB}$ \\
\hline Audiogram 4 right ear & Mild HL in all freq & $3.41 \mathrm{~dB}$ & $3.19 \mathrm{~dB}$ & $2.41 \mathrm{~dB}$ & $2.32 \mathrm{~dB}$ \\
\hline Audiogram 5 left ear & Moderate HL in all freq & $2.98 \mathrm{~dB}$ & $2.87 \mathrm{~dB}$ & $2.85 \mathrm{~dB}$ & $2.37 \mathrm{~dB}$ \\
\hline Audiogram 5 right ear & Moderate $\mathrm{HL}$ in all freq & $3.26 \mathrm{~dB}$ & $3.08 \mathrm{~dB}$ & $2.79 \mathrm{~dB}$ & $2.84 \mathrm{~dB}$ \\
\hline Audiogram 6 left ear & Profound HL in all freq & $2.43 \mathrm{~dB}$ & $2.39 \mathrm{~dB}$ & $2.62 \mathrm{~dB}$ & $2.31 \mathrm{~dB}$ \\
\hline Audiogram 6 right ear & Profound HL in all freq & $2.22 \mathrm{~dB}$ & $2.62 \mathrm{~dB}$ & $2.41 \mathrm{~dB}$ & $2.38 \mathrm{~dB}$ \\
\hline
\end{tabular}

in the design. The proposed linear phase FIR filter with 35 coefficients can easily be implemented using 18 multipliers. The hardware complexity of different filter bank generation methods for audiogram matching applications is estimated and listed in Table 8 along with the design specifications. The octave and fractional interpolation procedures do not 


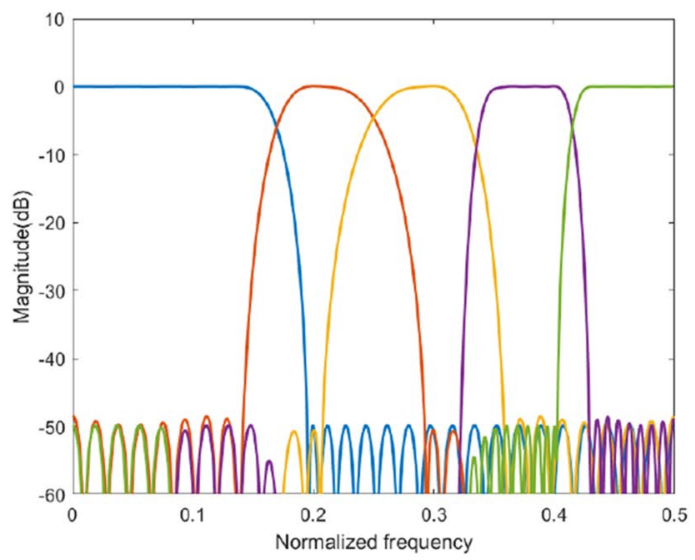

(a) Magnitude response of the filter bank for Audiogram 1 left ear.

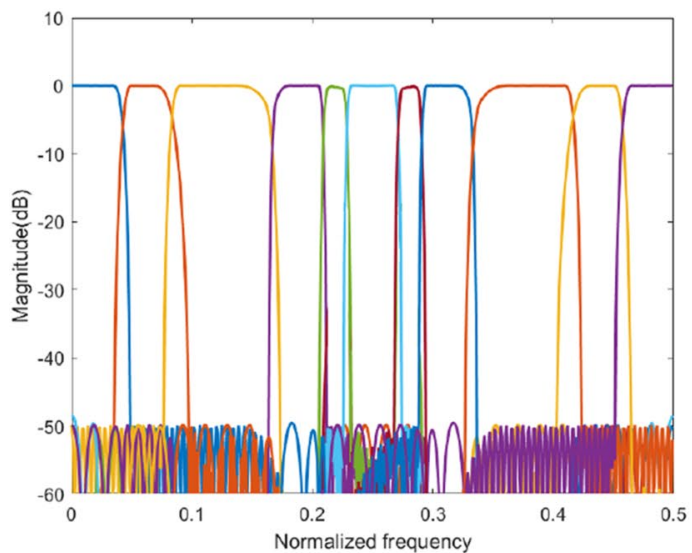

(c) Magnitude response of the filter bank for Audiogram 3 left ear.

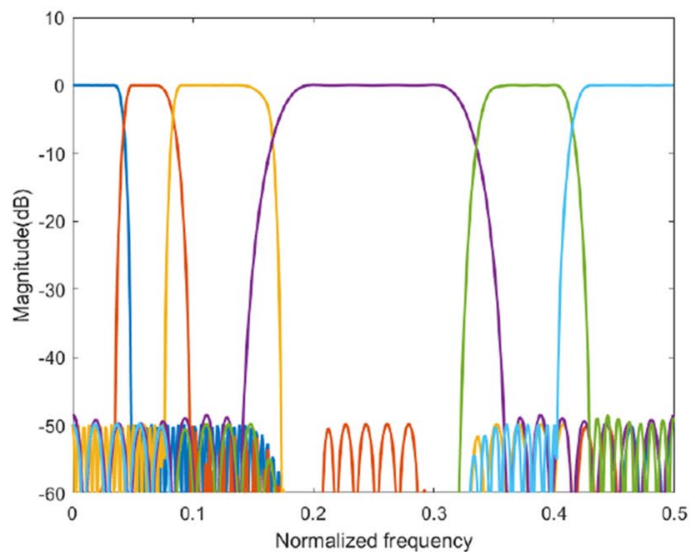

(e) Magnitude response of the filter bank for Audiogram 5 right ear.
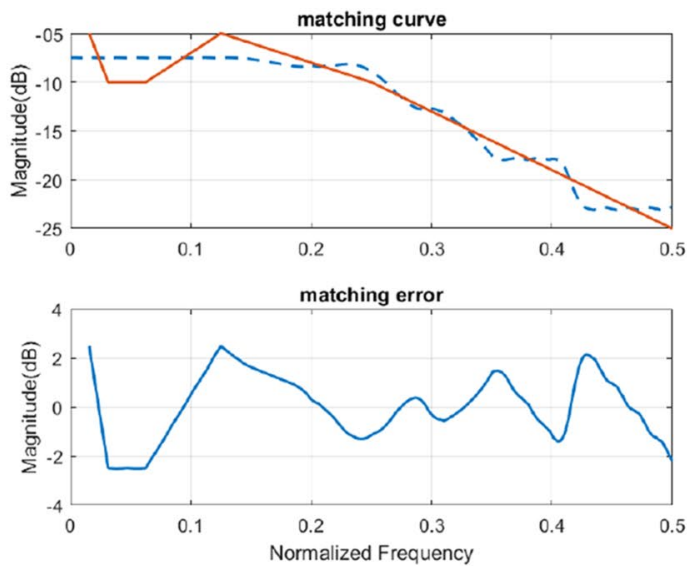

(b) Matching results for Audiogram 1 left ear.
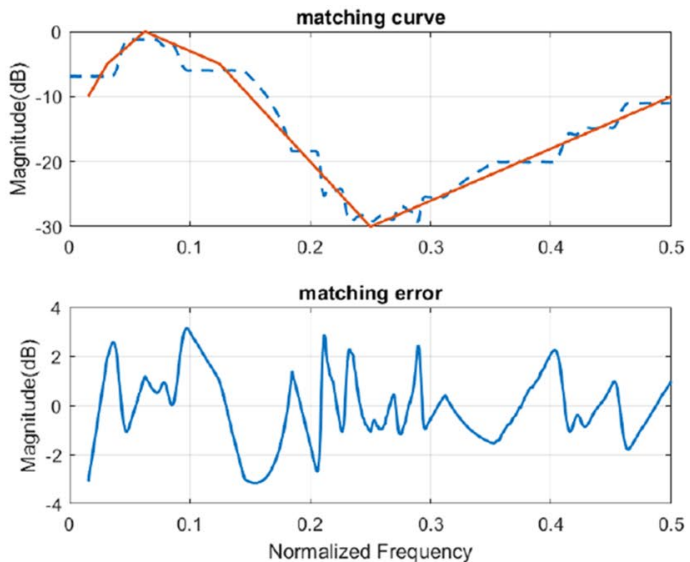

(d) Matching results for Audiogram 3 left ear.
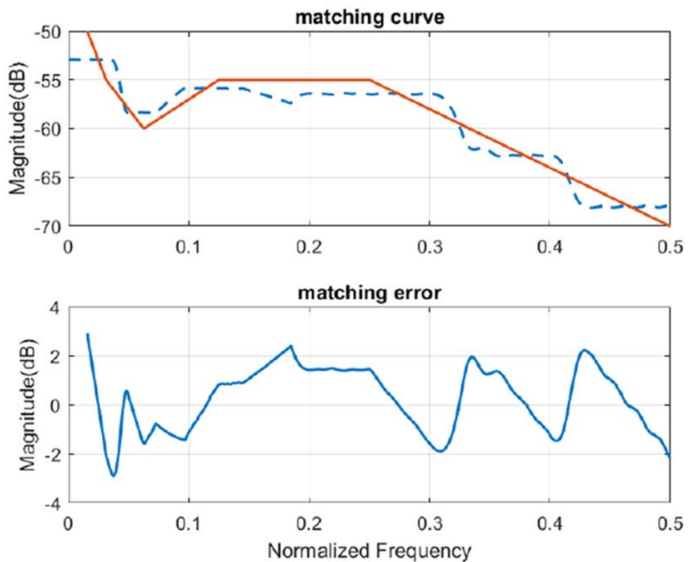

(f) Matching results for Audiogram 5 right ear.

Fig. 5 Magnitude responses of the filter banks with optimum scheme and the matching results 
Table 7 Scheme selection and maximum delay in audiogram matching

\begin{tabular}{lllll}
\hline Test audiogram & Region 1 & Region 2 & Region 3 & Delay (ms) \\
\hline $\begin{array}{l}\text { Audiogram 1 left ear } \\
\text { Audiogram 1 right }\end{array}$ & Scheme 1 & Scheme 2 & Scheme 2 & 4.00 \\
ear & Scheme 1 & Scheme 1 & Scheme 1 & 1.09 \\
Audiogram 2 left ear & Scheme 2 & Scheme 1 & Scheme 2 & 4.00 \\
Audiogram 2 right & Scheme 2 & Scheme 2 & Scheme 1 & 4.00 \\
ear & & & & \\
Audiogram 3 left ear & Scheme 3 & Scheme 4 & Scheme 3 & 18.54 \\
Audiogram 3 right & Scheme 3 & Scheme 4 & Scheme 4 & 18.54 \\
ear & & & & \\
Audiogram 4 left ear & Scheme 3 & Scheme 1 & Scheme 2 & 8.36 \\
Audiogram 4 right & Scheme 3 & Scheme 2 & Scheme 1 & 8.36 \\
ear & & & & \\
Audiogram 5 left ear & Scheme 3 & Scheme 2 & Scheme 1 & 8.36 \\
Audiogram 5 right & Scheme 3 & Scheme 1 & Scheme 2 & 8.36 \\
ear & & & & \\
Audiogram 6 left ear & Scheme 2 & Scheme 1 & Scheme 1 & 4.00 \\
Audiogram 6 right & Scheme 2 & Scheme 1 & Scheme 1 & 4.00 \\
ear & & & & \\
\hline
\end{tabular}

increase the number of multipliers. Table 8 also illustrates the percentage of reduction in complexity of the proposed structure with other structures. The complexity reduction percentage is the amount of reduction in coefficient multipliers in the proposed method with respect to other reported techniques. Let $N_{1}$ be the number of multipliers used in the existing technique and $N_{2}$ be the number of multipliers in the proposed method. The complexity reduction percentage is calculated using Eq. 7.

Complexity reduction percentage $=\left(\frac{N_{1}-N_{2}}{N_{1}}\right) \times 100$

For example, the complexity reduction percentage of the proposed method with respect to the Quasi-ANSI S1.11 filter bank technique is $((226-18) / 226) \times 100=92 \%$. The proposed method is capable of reducing the hardware complexity from a minimum of $40 \%$ to a maximum of $92 \%$ than the existing methods. Hence, the hardware implementation is possible with fewer components and small device area, thereby accomplishing cost-effective hearing aids of minimal complexity. Figure 6 gives a detailed comparison between the hardware complexity and delay of various auditory compensation techniques with the proposed method. The proposed method has the optimum delay and complexity when compared to other methods.

\section{Hardware implementation advantages}

The hardware implementation diagram of the proposed reconfigurable filter bank structure is shown in Fig. 7. A linear phase FIR filter of odd length with symmetric coefficients $\left[h_{(N-1) / 2}, \ldots, h_{1}, h_{0}, h_{1}, \ldots, h_{(N-1) / 2}\right]$, is selected as the prototype filter $H_{l}(z)$ of order $N$. The transposed form based design of the FIR filter is used to share the multipliers for symmetric coefficients. This design technique benefits a

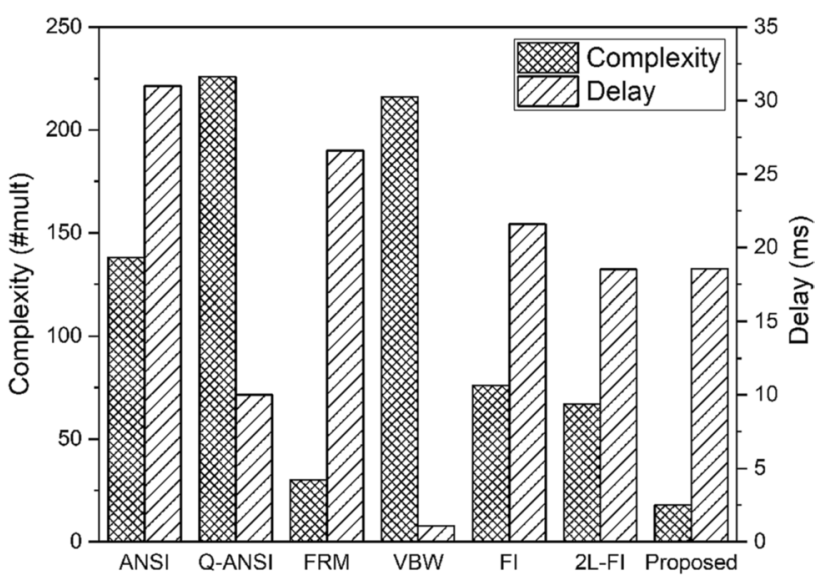

Fig. 6 Comparison of auditory compensation methods
Table 8 Comparison of complexity of the proposed structure with existing methods

\begin{tabular}{lllcll}
\hline Audiogram matching method & $f_{s}(\mathrm{kHz})$ & $\delta_{p}(\mathrm{~dB})$ & $\delta_{s}(\mathrm{~dB})$ & $\begin{array}{l}\text { Total no. of mul- } \\
\text { tipliers (\#mult) }\end{array}$ & $\begin{array}{l}\text { Complexity } \\
\text { reduction per- } \\
\text { centage }\end{array}$ \\
\hline ANSI S1.11 Filter bank [13] & 24 & 1 & 60 & 138 & $86.9 \%$ \\
Quasi-ANSI S1.11 Filter bank [14] & 24 & 1 & 60 & 226 & $92.0 \%$ \\
Frequency Response Masking [16] & 16 & 0.0001 & 80 & 30 & $40.0 \%$ \\
Cosine Modulation [19] & 16 & 0.01 & 110 & 63 & $71.4 \%$ \\
Variable Bandwidth Filters [22] & 16 & 0.05 & 80 & 216 & $91.6 \%$ \\
Modified DFT [23] & 16 & 0.001 & 85 & 84 & $78.5 \%$ \\
Fractional Interpolation (FI) [24] & 16 & 0.005 & 50 & 76 & $76.3 \%$ \\
Two-level FI [25] & 16 & 0.005 & 50 & 67 & $73.1 \%$ \\
Proposed method & 16 & 0.05 & 50 & 18 & -
\end{tabular}


Fig. 7 Hardware implementation of the proposed octave and fractional interpolated filters. (a) Hardware implementation of octave interpolated filters. (b) Hardware implementation of fractional interpolated filters

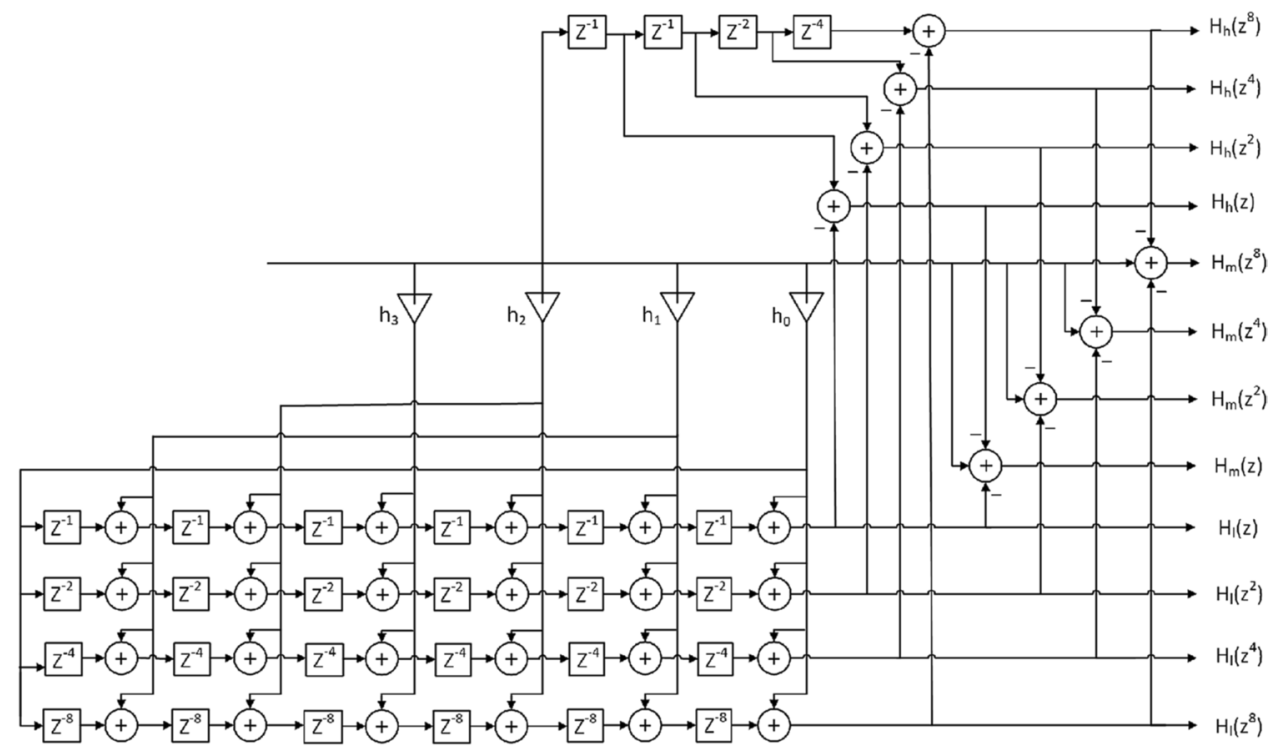

(a) Hardware implementation of octave interpolated filters.

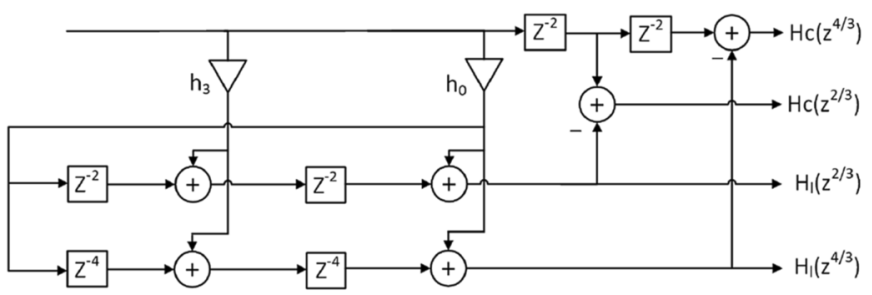

(b) Hardware implementation of fractional interpolated filters. reduction in hardware complexity and device utilization. In Fig. 7, a 7-tap prototype filter with symmetric coefficients $\left[h_{3}, h_{2}, h_{1}, h_{0}, h_{1}, h_{2}, h_{3}\right]$ is chosen for the hardware implementation of the octave and fractional interpolated filters. The prototype filter, $H_{l}(z)$ is implemented using a single delay element between each adder. $L$ delay elements are inserted between the adders to obtain the $L$-fold interpolated filter, $H_{l}\left(z^{L}\right)$. Since the number of multipliers is not changed, the complexity of the device will not be increased with interpolation.

The design and evaluation of the proposed system are performed using the MATLAB R2017b simulation software with signal processing and filter design toolboxes. The hardware implementation of the system is evaluated in Verilog language using Xilinx Vivado 2018.3 software on Xilinx Kintex-7 FPGA development board. The implementation results of the proposed structure compared with similar structures in $[24,25]$ are shown in Table 9. Since the sampling rate of the proposed system is $16 \mathrm{kHz}$, the hardware uses the same clock frequency. From Table 9, it is evident that the device utilization and the power dissipation at $16 \mathrm{kHz}$ of the proposed structure are promising than other similar structures.

\section{Conclusion}

An auto-reconfigurable filter bank structure of reduced complexity for audiogram matching applications in a digital hearing aid is discussed in this paper. Multi-branch
Table 9 Device and power utilization

\begin{tabular}{llll}
\hline Utilization data & Structure in [24] & Structure in [25] & $\begin{array}{l}\text { Proposed } \\
\text { structure }\end{array}$ \\
\hline Number of slice registers & 5376 & 4557 & 3016 \\
Number of LUTs & 13092 & 11511 & 7985 \\
Number of FF pairs & 4160 & 3378 & 2467 \\
Power at 16 kHz (Watt) & 0.470 & 0.470 & 0.398 \\
\hline
\end{tabular}


frequency response masking technique based on the octave and fractional interpolations is used to decompose the audio spectrum into several sub-bands. The proposed ParksMcClellan based prototype FIR filter has only 18 coefficient multipliers, thereby demanding only a minimal chip area for its implementation. This proposed structure also makes the hearing aid more compact and power-efficient. There is a massive reduction of multipliers up to $92 \%$ when compared to other existing techniques. This auto-reconfigurable filter bank structure decomposes the audio spectrum into three regions. An optimum scheme selection method is proposed in each region based on the sharpness of hearing profile in the audiogram. The same structure can be used for various types of hearing losses by simply adjusting the control switches. The matching errors and the operational delays of the proposed structure are within the globally accepted limits. This proposed filter bank structure integrated with automatic reconfiguring capability can effectively help in reducing the tiresome manual interventions of adjusting the proper bands in the respective positions in the hearing aid. This method of reduced complexity can also help in minimizing the hardware requirements which can ultimately result in realizing the vision of affordable hearing aids to the needy.

Acknowledgements This work is funded by the Centre for Engineering Research and Development (CERD) of APJ Abdul Kalam Technological University, India (Grant No: KTU/A/199/2016).

\section{Declarations}

Conflict of interest The authors declare that they have no conflict of interest.

Ethical approval For this type of study, formal consent is not required.

Informed consent This article does not contain any studies with human participants or animals performed by any of the authors.

\section{References}

1. Levitt H (2007) A historical perspective on digital hearing aids: how digital technology has changed modern hearing aids. Trends Amplif 11(1):7-24

2. Bentler R, Mueller HG, Ricketts TA (2016) Modern hearing aids: Verification, outcome measures, and follow-up. Plural Publishing

3. Kar A, Anand A, Østergaard J, Jensen SH, Swamy MNS (2019) Sound quality improvement for hearing aids in presence of multiple inputs. Circuits Syst Signal Process 38(8):3591-3615

4. Yu-Ting K, Tay-Jyi L, Yueh-Tai L, Wei-Han C, Chih-Wei L, Shuenn-Tsong Y (2007) Design of ANSI S1. 11 filter bank for digital hearing aids. In 2007 14th IEEE International Conference on Electronics, Circuits and Systems, IEEE, pp 242-245

5. Wei Y, Ma T, Ho BK, Lian Y (2018) The design of low-power 16-band nonuniform filter bank for hearing aids. IEEE Trans Biomed Circuits Syst 13(1):112-123
6. Kilıc O, Kalcıglu MT, Cag Y, Tuysuz O, Pektas E, Caskurlu H, Cetın F (2020) Could sudden sensorineural hearing loss be the sole manifestation of covid-19? an investigation into sars-cov-2 in the etiology of sudden sensorineural hearing loss. Int J Infect Dis 97:208-211

7. Khullar S, Babbar R (2011) Presbycusis and auditory brainstem responses: a review. Asian Pac J Trop Dis 1(2):150-157

8. WHO (2017) Preferred profile for hearing-aid technology suitable for low-and middle-income countries. World Health Organization

9. McPherson B (2018) Hearing aid systems in low- resource settings. Community Ear Hear Health J 15(19):1-2

10. Bruce Tomblin J, Oleson JJ, Ambrose SE, Walker E, Moeller MP (2014) The influence of hearing aids on the speech and language development of children with hearing loss. JAMA OtolaryngolHead Neck Surg 140(5):403-409

11. Mitchell BL, Thorp Jackson G, Evans David M, Nyholt Dale R, Martin Nicholas G, Lupton Michelle K (2020) Exploring the genetic relationship between hearing impairment and Alzheimer's disease. Alzheimer's Dement Diagn Assess Dis Monitor 12(1):e12108

12. Lin FR, Ferrucci L, Yang An JO, Goh JD, Metter EJ, Davatzikos C, Kraut MA, Resnick SM (2014) Association of hearing impairment with brain volume changes in older adults. Neuroimage 90:84-92

13. Kuo Y-T, Lin T-J, Li Y-T, Liu C-W (2009) Design and implementation of low-power ANSI S1. 11 filter bank for digital hearing aids. IEEE Trans Circuits Syst I: Regul Papers 57(7):1684-1696

14. Liu C-W, Chang K-C, Chuang M-H, Lin C-H (2012) 10-ms 18-band quasi-ANSI S1. 11 1/3-octave filter bank for digital hearing aids. IEEE Trans Circuits Syst I: Regul Papers 60(3):638-649

15. Manju M, Elizabeth E (2011) Design of multiplier-less FRM FIR filter using artificial bee colony algorithm. In: 2011 20th European Conference on Circuit Theory and Design (ECCTD), IEEE, pp 322-325

16. Lian Y, Wei Y (2005) A computationally efficient nonuniform FIR digital filter bank for hearing aids. IEEE Trans Circuits Syst I: Regul Papers 52(12):2754-2762

17. Bindiya TS, Elias E (2014) Metaheuristic algorithms for the design of multiplier-less non-uniform filter banks based on frequency response masking. Soft Comput 18(8):1529-1547

18. Manuel M, Elias E (2013) Design of frequency response masking FIR filter in the canonic signed digit space using modified artificial bee colony algorithm. Eng Appl Artif Intel 26(1):660-668

19. Kalathil S, Elias E (2015) Efficient design of non-uniform cosine modulated filter banks for digital hearing aids. AEU-Int J Electron Commun 69(9):1314-1320

20. Deng T-B (2010) Three-channel variable filter-bank for digital hearing aids. IET signal process 4(2):181-196

21. Indrakanti R, Elias E (2019) Design of low-complexity farrow structure-based reconfigurable filters for parallel spectrum hole detection. Signal Image Video Process 13(4):787-794

22. Haridas N, Elias E (2016) Efficient variable bandwidth filters for digital hearing aid using farrow structure. J Adv Res 7(2):255-262

23. Vellaisamy S, Elias E (2018) Design of hardware-efficient digital hearing aids using non-uniform MDFT filter banks. Signal Image Video Process 12(8):1429-1436

24. Wei Y, Wang Y (2015) Design of low complexity adjustable filter bank for personalized hearing aid solutions. IEEE/ACM Trans Audio Speech Language Process 23(5):923-931

25. Amir A, Bindiya TS, Elias Elizabeth (2018) Design and implementation of reconfigurable filter bank structure for low complexity hearing aids using 2-level sound wave decomposition. Biom Signal Process Control 43:96-109

26. Tomson D, Manju M (2018) A 17-band non-uniform interpolated FIR filter bank for digital hearing aid. In 2018 International 
conference on communication and signal processing (ICCSP), IEEE, pp 0452-0456

27. Devis T, Manuel M (2020) A low-complexity 3-level filter bank design for effective restoration of audibility in digital hearing aids. Biomed Eng Lett 10(4):593-601

28. Oppenheim AV (1999) Discrete-time signal processing. Pearson Education India

29. McClellan J, Parks TW, Rabiner L (1973) A computer program for designing optimum FIR linear phase digital filters. IEEE Trans Audio Electroacoust 21(6):506-526

30. Mitra SK, Kuo Y (2006) Digital signal processing: A computerbased approach, vol 2. McGraw-Hill, New York

31. Vaidyanathan PP (2006) Multirate systems and filter banks. Pearson Education India

32. Devis T, Manuel M (2018) Multirate and filterbank approaches in digital hearing aid design: A review. In IOP Conference Series: Materials Science and Engineering, vol 396. IOP Publishing, pp 012036.
33. Vogel DA, McCarthy PA, Bratt GW, Brewer C (2007) The clinical audiogram: its history and current use. Commun Disord Rev $1(2): 81-94$

34. Rüdiger K, Klaus-Peter H, Steven PR (2011) Springer handbook of medical technology. Springer Science \& Business Media

35. Clark JG (1981) Uses and abuses of hearing loss classification. ASHA 23(7):493-500

36. Walker JJ, Cleveland LM, Davis JL, Seales JS (2013) Audiometry screening and interpretation. Am Fam Phys 87(1):41-47

37. Consumer resource for hearing aids. [online]. http://www.earinfo. com/how-to-read-a-hearing-aid-test/. Accessed 10 July 2020

Publisher's Note Springer Nature remains neutral with regard to jurisdictional claims in published maps and institutional affiliations. 